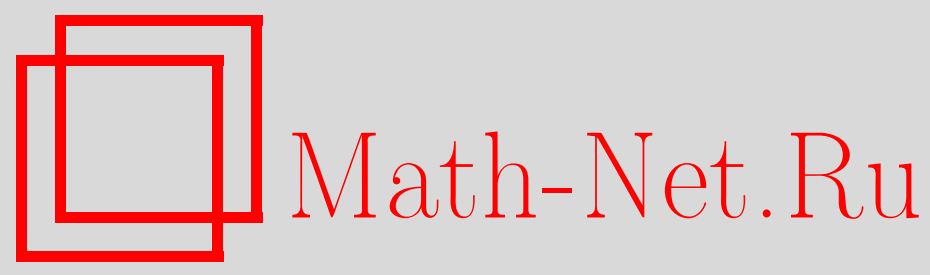

Ю. Г. Леонов, Проблема сопряженности в одном классе 2групп, Матем. заметки, 1998, том 64, выпуск 4, 573-583

DOI: https://doi.org/10.4213/mzm1432

Использование Общероссийского математического портала Math-Net.Ru подразумевает, что вы прочитали и согласны с пользовательским соглашением http://www.mathnet.ru/rus/agreement

Параметры загрузки:

IP : 54.147 .182 .235

26 апреля 2023 г., 16:12:48

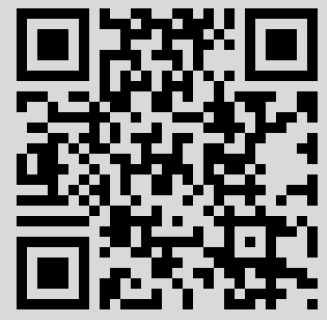




\title{
ПРОБЛЕМА СОПРЯЖЕННОСТИ В ОДНОМ КЛАССЕ 2-ГРУПП
}

\author{
Ю.Г. Леонов
}

В работе разрешена проблема сопряженности элементов в 2-группах Григорчука. Каждая группа рассматривается с позиции преобразований интервала $(0,1)$ типа "перекладьваний" и с позиции сплетений групп. Указано несколько подходов, позволяющих выяснить сопряженность элементов.

Библиограффия: 5 названий.

1. Введение. В 1980 году Р. И. Григорчук построил новый замечательньй пример бесконечной 3-порожденной 2-групшы [1]. Впоследствии он обобщил свою конструкцию, получив класс не локально конечных 2-груп, обладающих дополнительно цельм рядом интересных свойств. Каждая из этих групп является финитно-аппоксимируемой, аменабельной, имеет промежуточньй рост [2].

Определим групшы данного класса, следуя [2]. Рассмотрим пространство последовательностей

$$
\Omega=\left\{\omega=\left\{\omega_{n}\right\}_{n=1}^{\infty}, \omega_{n} \in\{1,2,3\}\right\} .
$$

Зададим отображение $\omega_{i} \mapsto \bar{\omega}_{i}$, где

$$
\overline{1}=(T, \Pi, \Pi), \quad \overline{2}=(\Pi, T, \Pi), \quad \overline{3}=(\Pi, \Pi, T),
$$

$T, \Pi$ - буквы. Пусть $W_{1, \omega}, W_{2, \omega}, W_{3, \omega}$ - бесконечные слова в алфавите $\{T, \Pi\}$, совпадающие соответственно с первой, второй и третьей строками в последовательности $\bar{\omega}=\bar{\omega}_{1} \bar{\omega}_{2} \ldots \bar{\omega}_{n} \ldots$ Определим преобразования интервала $(0,1)$, взятого без точек вида $k / 2^{n}$, где $n \in \mathbb{Z}_{+}, k=\overline{0,2^{n}}$ :

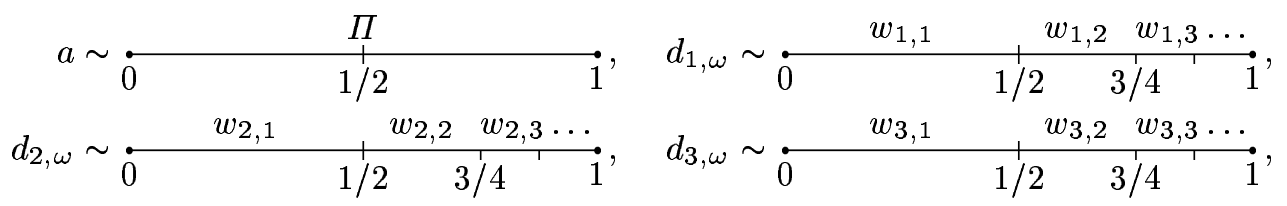

где

$$
W_{1, \omega}=w_{1,1} \ldots w_{1, n} \ldots, \quad W_{2, \omega}=w_{2,1} \ldots w_{2, n} \ldots, \quad W_{3, \omega}=w_{3,1} \ldots w_{3, n} \ldots .
$$

Под буквами $T$ и $\Pi$ будем понимать соответственно тождественное преобразование и перестановку половин интервала, к которому эта буква относится. Таким образом, по определению буквы $T$ и $\Pi$ относятся к интервалам вида $\left(1-1 / 2^{n}, 1-1 / 2^{n+1}\right), n \in \mathbb{Z}_{+}$. Группу $G_{\omega}=\left\langle a, d_{1, \omega}, d_{2, \omega}, d_{3, \omega}\right\rangle$ назовем группой Григорчука типа $\omega$. 
Согласно [2] $G_{\omega}$ является бесконечной, финитно-аппроксимируемой 2-группой при $\omega \in \Omega_{0} \subset \Omega$, где $\Omega_{0}-$ пространство последовательностей $\omega$, в которые цифры $1,2,3$ входят бесконечное число раз. В группе $G_{\omega}$ разрешима проблема равенства тогда и только тогда, когда $\omega$ - рекурсивная последовательность [2]. Однако, вопрос о разрешимости проблемы сопряженности до сих пор оставался открытым.

Пусть $R \Omega_{0}=\left\{\omega \in \Omega_{0}, \omega\right.$ - рекурсивная последовательность $\}, R_{1} \Omega_{0}=\left\{\omega \in R \Omega_{0}\right.$, $\left.\omega_{n} \neq \omega_{n+1}, n \in \mathbb{N}\right\}$. Целью настоящей работы является доказательство следующего результата.

Теорема. B әруппе $G_{\omega}, \omega \in R_{1} \Omega_{0}$, разрешима проблема сопряженности.

Предложенньй нами метод объединяет два подхода исследований группы Григорчука с позиции преобразований интервала $(0,1)$ типа “перекладываний” и с позиции сплетений групп. Последнее является следствием того, что группа $G_{\omega}$ есть подгруппа сплетения $P_{\infty}=l_{i=1}^{\infty} \mathbb{Z}_{2}^{(i)}$. Каждый элемент такого сплетения может быть задан так назьваемой “таблицей" [3] вида

$$
\left[b_{0}, b_{1}\left(x_{1}\right), b_{2}\left(x_{1}, x_{2}\right), \ldots, b_{k}\left(x_{1}, \ldots, x_{k}\right), \ldots\right]
$$

где $x_{1}, x_{2}, \ldots$ - формальные переменные кольца $\mathbb{Z}_{2}, b_{0}, b_{1}, \ldots$ - полиномы от соответствующих переменных с коэффициентами из кольца $\mathbb{Z}_{2}$. Произведение таблищ определим следуюшим образом:

$$
\begin{aligned}
& {\left[b_{0}, b_{1}\left(x_{1}\right), b_{2}\left(x_{1}, x_{2}\right), \ldots\right] \cdot\left[c_{0}, c_{1}\left(x_{1}\right), c_{2}\left(x_{1}, x_{2}\right), \ldots\right]} \\
& \quad=\left[b_{0}+c_{0}, b_{1}\left(x_{1}\right)+c_{1}\left(x_{1}+b_{0}\right), b_{2}\left(x_{1}, x_{2}\right)+c_{2}\left(x_{1}+b_{0}, x_{2}+b_{1}\left(x_{1}\right)\right), \ldots\right]
\end{aligned}
$$

В этих терминах порождающие элементы групы $G_{\omega}$ можно представить так:

$$
a=[1,0,0, \ldots], \quad d_{j, \omega}=\left[0, T_{j}^{1}\left(x_{1}+1\right), \ldots, T_{j}^{k}\left(x_{1} x_{2} \cdots x_{k-1}\left(x_{k}+1\right)\right), \ldots\right],
$$

где

$$
T_{j}^{k} Y=\left\{\begin{array}{l}
0, \text { если } j=\omega_{k}, \\
Y, \quad \text { если } j \neq \omega_{k},
\end{array} \quad k \in \mathbb{N},\right.
$$

а $Y$ - любой многочлен.

2. Проекции. Элементы группы $G_{\omega}$ имеют сложное внутреннее строение. Поэтому для получения более полной информации о действии $g \in G_{\omega}$ на интервал $(0,1)$ имеет смысл рассматривать сужения преобразований на подынтервалах. Будем назьвать

$$
R_{n}^{(i)} \stackrel{\text { def }}{=}\left(\frac{i-1}{2^{n}}, \frac{i}{2^{n}}\right), \quad \text { где } i=\overline{1,2^{n}}, \quad n \in \mathbb{Z}_{+},
$$

интервалом уровня $n$.

Пусть $H_{\omega}^{(n)}$ - подгрупша групшы $G_{\omega}$, состоящая из элементов, переводящих интервалы уровня $n$ в себя. Таким образом, каждый элемент $g \in H_{\omega}^{(n)}$ определяет на интервале $R_{n}^{(i)}$ некоторое преобразование $\varphi_{R_{n}^{(i)}}(g)$, которое назовем проекиией $g$ на $R_{n}^{(i)}$. Заметим (cp. [2]), что проекции элементов группы $H_{\omega}^{(n)}$ на интервалы уровня $n$ образуют группу $G_{\sigma^{n}} \omega$, где $\sigma$ - сдвиг влево в пространстве последовательностей: $\sigma \omega=\left\{\omega_{2}, \omega_{3}, \ldots\right\}$, а $\sigma^{n}$ есть $n$-кратное повторение сдвига: $\sigma^{n} \omega=\left\{\omega_{n+l}\right\}_{l=1}^{\infty}$. 
Считая $\omega$ фиксированной последовательностью пространства $R_{1} \Omega_{0}$, для упрощения обозначений в этом пунктеположим $d_{j}=d_{j, \omega}, G_{n}=G_{\sigma^{n}} \omega, H_{n}=H_{\sigma^{n}}{ }, G=G_{0}=G_{\omega}$, $H^{(n)}=H_{\omega}^{(n)}$. Пусть $\Phi=\{1,2,3\}$. Порождаюшие элементы групшы $G_{n}$ обозначим через $a_{n},\left(d_{j}\right)_{n}$, где $j \in \Phi, n \in \mathbb{Z}_{+}$.

В группе $G$ очевидны следуюшие соотношения:

$$
a^{2}=d_{j_{1}}^{2}=1, \quad d_{j_{1}} d_{j_{2}}=d_{j_{3}},
$$

где $j_{1}, j_{2}, j_{3}$ - попарно различные элементы множества $\Phi$. Следовательно, элементы $d_{1}$, $d_{2}$ порождают групу $\left\{1, d_{1}, d_{2}, d_{3}\right\} \cong \mathbb{Z}_{2} \times \mathbb{Z}_{2}$. Единицу этой группы иногда будем обозначать через $d_{0}$.

Группа $H=H^{(1)}=\left\langle d_{1}, d_{2}, d_{3}, a d_{1} a, a d_{2} a, a d_{3} a\right\rangle$ имеет следующую таблицу проектирования своих порождающих:

\begin{tabular}{|c|c|c|c|c|c|c|}
\hline & $d_{1}$ & $d_{2}$ & $d_{3}$ & $a d_{1} a$ & $a d_{2} a$ & $a d_{3} a$ \\
\hline$\varphi_{R_{1}^{(1)}}$ & $u_{1,1}$ & $u_{2,1}$ & $u_{3,1}$ & $\left(d_{1}\right)_{1}$ & $\left(d_{2}\right)_{1}$ & $\left(d_{3}\right)_{1}$ \\
\hline$\varphi_{R_{1}^{(2)}}$ & $\left(d_{1}\right)_{1}$ & $\left(d_{2}\right)_{1}$ & $\left(d_{3}\right)_{1}$ & $u_{1,1}$ & $u_{2,1}$ & $u_{3,1}$ \\
\hline
\end{tabular}

где

$$
u_{j, n}= \begin{cases}a_{n}, & \text { если } j \neq \omega_{n}, \\ 1, & \text { если } j=\omega_{n} .\end{cases}
$$

Аналогичную операцию проектирования можно произвести и в терминах сплетений. Если $g=\left[0, b_{1}\left(x_{1}\right), b_{2}\left(x_{1}, x_{2}\right), \ldots\right] \in H$, то

$$
\varphi_{R_{1}^{(1)}}(g)=\left[b_{1}(0), b_{2}\left(0, x_{1}\right), \ldots\right], \quad \varphi_{R_{1}^{(2)}}(g)=\left[b_{1}(1), b_{2}\left(1, x_{1}\right), \ldots\right]
$$

т.е. при проектировании таблицы на левьй (или правьй) полуинтервал осуществляется гомоморфизм формальных переменных

$$
\left.x_{1} \mapsto 0 \text { (или } 1\right), \quad x_{n} \mapsto x_{n-1} \text { при } n>1,
$$

а также сдвиг последовательности полиномов влево

$$
\left(0, b_{1}, b_{2}, \ldots\right) \mapsto\left(b_{1}, b_{2}, b_{3}, \ldots\right)
$$

При $g=\left[0, \ldots, 0, b_{n}\left(x_{1}, \ldots, x_{n}\right), \ldots\right] \in H^{(n)}$ проекция на любой интервал производится индуктивно с использованием проектирования на один шаг.

В общем случае $g \in G_{\omega}$ обычная проекция не осуществима, однако при помощи сплетения можно определить аналог проекции на интервалы уровня $n \in \mathbb{N}$ :

$$
\varphi_{R_{n}^{(i)}}\left(\left[b_{0}, b_{1}\left(x_{1}\right), \ldots, b_{n}\left(x_{1}, \ldots, x_{n}\right), \ldots\right]\right)=\varphi_{R_{n}^{(i)}}\left(\left[0, \ldots, 0, b_{n}\left(x_{1}, \ldots, x_{n}\right), \ldots\right]\right) .
$$

При этом элемент $g^{(n-1)}=\left[b_{0}, b_{1}\left(x_{1}\right), \ldots, b_{n-1}\left(x_{1}, \ldots, x_{n-1}\right)\right]$ назовем верхней частью $g$ уровня $n-1$. 
При фиксированном $n$ рассмотрим $g_{i}=\varphi_{R_{n}^{(i)}}(g)$. Набор $s_{n}(g)=\left(g_{1}, \ldots, g_{2^{n}} ; g^{(n-1)}\right)$ назовем схемой әлемента $g$ уровня $n$, а последовательность $\partial_{n}(g)=\left(g_{1}, \ldots, g_{2^{n}}\right)$ - гpaницей элемента $g$ уровня $n$. Очевидно, что каждый элемент $g \in G$ однозначно задает схему любого уровня. Так как у разных элементов таблицы различны, $s_{n}(g) \neq s_{n}(h)$ при $g \neq h$.

Заметим, что группа $G$ финитно аппроксимируется групшами

$$
K^{(n)}=\left\langle a, d_{1}^{(n)}, d_{2}^{(n)}\right\rangle \leqslant P_{n+1}=2_{i=1}^{n+1} \mathbb{Z}_{2}^{(i)} \leqslant S_{2^{n+1}},
$$

где $S_{2^{n+1}}$ - симметрическая группа степени $2^{n+1}$.

Пусть $s_{n}(g)=\left(g_{1}, \ldots, g_{2^{n}} ; g^{(n-1)}\right), s_{n}(h)=\left(h_{1}, \ldots, h_{2^{n}} ; h^{(n-1)}\right)$. Из сказанного выше о представлении элементов $g$ и $h$ в виде элементов бесконечного сплетения, а элементов $g^{(n-1)}$ и $h^{(n-1)}$ в виде конечного сплетения имеем

$$
s_{n}(g h)=\left(g_{1} h_{\rho 1}, \ldots, g_{2^{n}} h_{\rho 2^{n}} ; g^{(n-1)} h^{(n-1)}\right) .
$$

Здесь $\rho=\left(g^{(n-1)}\right)^{-1}$ действует на множество $\left\{1, \ldots, 2^{n}\right\}$ как элемент группы $S_{2^{n}}$.

Из (2) видно, что длины проекций элемента $g$ при проектировании всегда меньше длины $g$, кроме случая, когда $g$ - порождаюший или единичньй элемент. Отсюда следует, что существует $n \in \mathbb{N}$ такое, что элементы последовательности $\partial_{n}(g)$ принадлежат множеству $\left\{1, a_{n},\left(d_{1}\right)_{n},\left(d_{2}\right)_{n},\left(d_{3}\right)_{n}\right\}$. Наименьшее $n$ с таким свойством назовем рангом әлемента $g$ и обозначим через $f(g)$. Положим $f(a)=f(1)=f\left(d_{j}\right)=0, j \in \Phi$.

Специфика действия элемента $g \in G$ на интервале $(0,1)$ влечет необходимость ряда определений на язьке подьнтервалов.

Дополнением к интервалу $R_{n}^{(k)}$ или связанным с ним интервалом первого уровня назовем интервал $\Delta_{1} R_{n}^{(k)}=R_{n}^{(\delta(k))}$, где

$$
\delta(k)= \begin{cases}k+1, & \text { если } k \text { нечетное, } \\ k-1, & \text { если } k \text { четное. }\end{cases}
$$

Таким образом, вьполняется $R_{n}^{(k)} \cup \Delta_{1} R_{n}^{(k)}=R_{n-1}^{[(k+1) / 2]}$. По индукции $i$-м дополнением или связанным интервалом $i$-го уровня $(i>1)$ для $R_{n}^{(k)}(n>1)$ назовем интервал

$$
\Delta_{i} R_{n}^{(k)}=\Delta_{1}\left(\Delta_{i-1} R_{n}^{(k)} \cup \Delta_{1}\left(\Delta_{i-1} R_{n}^{(k)}\right)\right) .
$$

Положим ind $\left(\left(d_{j}\right)_{n}\right)=j, \operatorname{ind}\left(1_{G_{n}}\right)=\operatorname{ind}\left(a_{n}\right)=0$.

Пусть $t=\left(a_{n}\right)\left(d_{j_{1}}\right)_{n} a_{n}\left(d_{j_{2}}\right)_{n} \ldots a_{n}\left(d_{j_{\mu}}\right)_{n}\left(a_{n}\right)$. Запись $\left(a_{n}\right) t$ (или $\left.t\left(a_{n}\right)\right)$ означает, что мы рассматриваем одновременно $t$ и $a_{n} t$ (или $t$ и $\left.t a_{n}\right)$. Индексом әлемента $t \in G_{n}$ назовем элемент $I(t)=\operatorname{ind}\left(\left(d_{j_{1}}\right)_{n}\left(d_{j_{2}}\right)_{n} \ldots\left(d_{j_{\mu}}\right)_{n}\right) \in \Phi$.

Для произвольного множества $X$ зададим функцию со значениями в кольце $\mathbb{Z}_{2}$ :

$$
Q(x, X)= \begin{cases}1, & \text { если } x \notin X, \\ 0, & \text { если } x \in X .\end{cases}
$$

Множество элементов вида $(\nu, \varepsilon, \tau), \nu \in\left\langle 1, d_{1}, d_{2}, d_{3}\right\rangle=\mathbb{Z}_{2} \times \mathbb{Z}_{2}, \varepsilon, \tau \in \mathbb{Z}_{2}$, превратим в группу $L_{y}, y \in \Phi$, определив произведение:

$$
\left(\nu_{1}, \varepsilon_{1}, \tau_{1}\right) \cdot\left(\nu_{2}, \varepsilon_{2}, \tau_{2}\right)=\left(\nu_{1}+\nu_{2}, \varepsilon_{1}+\varepsilon_{2}, \tau_{1}+\tau_{2}+\varepsilon_{1} \cdot Q\left(\nu_{2},\left\{1, d_{y}\right\}\right)\right) .
$$

Рассмотрим отображения

$$
\begin{gathered}
\varepsilon^{(n)}(t)=Q\left(t, H_{n}\right), \quad \tau^{(n)}(t)=Q\left(I\left(\varphi_{R_{1}^{(1)}}(t)\right),\left\{0, \omega_{n}\right\}\right) \\
\chi_{n}(t)=\left(d_{I(t)}, \varepsilon^{(n)}(t), \tau^{(n)}(t)\right) \in L_{\omega_{n}} .
\end{gathered}
$$


ПРЕДЛОЖЕНИЕ 1. Отображение $\chi_{n}$ является эпиморфизмом әруппы $G_{n}$ на $L_{\omega_{n}}$.

ДокаЗАтЕльство. Пусть $t_{1}, t_{2} \in G_{n}$. Докажем равенство $\chi_{n}\left(t_{1} t_{2}\right)=\chi_{n}\left(t_{1}\right) \cdot \chi_{n}\left(t_{2}\right)$.

Действительно, при $t=t_{1} t_{2}$ имеем $d_{I\left(t_{1}\right)} d_{I\left(t_{2}\right)}=d_{I(t)}$ и $\varepsilon^{(n)}(t)=Q\left(t_{1}, H_{n}\right)+Q\left(t_{2}, H_{n}\right)$. Осталось показать совпадение третьих координат. Если $\varepsilon_{1}=\varepsilon^{(n)}\left(t_{1}\right)=0$, то $\varphi_{R_{1}^{(1)}}(t)=$ $\varphi_{R_{1}^{(1)}}\left(t_{1}\right) \varphi_{R_{1}^{(1)}}\left(t_{2}\right)$. Следовательно, $\tau^{(n)}(t)=\tau_{1}+\tau_{2}$. В случае $\varepsilon_{1}=1$ выполнено

$$
I\left(\varphi_{R_{1}^{(1)}}(t)\right)=I\left(\varphi_{R_{1}^{(1)}}\left(t_{1}\right) \cdot \varphi_{R_{1}^{(2)}}\left(t_{2}\right)\right)=I\left(\varphi_{R_{1}^{(1)}}\left(t_{1}\right) \cdot \varphi_{R_{1}^{(1)}}\left(t_{2}\right) \cdot t_{2}\right)
$$

Отсюда $\tau^{(n)}(t)=\tau^{(n)}\left(t_{1}\right)+\tau^{(n)}\left(t_{2}\right)+Q\left(I\left(t_{2}\right),\left\{0, \omega_{n}\right\}\right)$.

Далее будем писать $\varepsilon=\varepsilon^{(n)}, \tau=\tau^{(n)}$, считая параметр $n$ определенньм по смыслу.

Элемент $\chi_{n}(t)$ назовем характеристикой $t \in G_{n}$ уровня $n$, а $L_{\omega_{n}}$ - әруппой характеристик этого уровня. Отображение $\tau$ иногда будем назьвать внутренним оператором.

Лемма 1. Пусть $t \in G_{n}, n \in \mathbb{Z}_{+}, u \chi_{n+1}\left(\varphi_{R_{1}^{(i)}}(t)\right)=\left(d_{j_{i}}, \varepsilon_{i}, \tau_{i}\right), i=1,2$. Тогдa

a) $\varepsilon_{i}=Q\left(j_{\delta(i)},\left\{0, \omega_{n+1}\right\}\right), i=1,2$;

б) $\tau_{1}+\tau_{2}=\varepsilon_{1} \cdot \varepsilon_{2}$.

ДокАЗАТЕЛЬСТво утверждений а) и б) проведем индукцией по длине слова $t$. База индукции для порождающих группы $G_{n}$ следует непосредственно из их определений.

Пусть len $(g)$ - длина элемента $g$ при заданной системе порождающих. Предположим, что лемма вьполнена для элементов длины меньшей $k$. Проектированием на половины интервала $(0,1)$ убеждаемся в верности утверждения для $t=t^{\prime} u$, где len $(t)=k$, $\operatorname{len}\left(t^{\prime}\right)=k-1$ и $u-$ один из порождаюших групшы $G_{n}$.

Для некоторого натурального $n$ рассмотрим произвольную последовательность $t_{1}$, $\ldots, t_{2^{n}}$ элементов групшы $G_{n}$. Очевидно, что в группе $P_{\infty}$ существует $\pi$ такое, что проекция таблищы $\varphi_{R_{n}^{(i)}}(\pi)$ равна $t_{i}, i=\overline{1,2^{n}}$.

Зафиксируем $t \in H_{n}$ и опишем процесс получения $\bar{t} \in H$ с условием $\varphi_{R_{n}^{\left(2^{n}\right)}}(\bar{t})=t$. При этом сушествует возможность выбрать $\bar{t}$, удовлетворяющим дополнительным свойствам (которые нам понадобятся в дальнейшем).

Лемма 2. а) Если $t \in H_{1}$, то әлемент $\bar{t}$ с проекиией $\varphi_{R_{1}^{(2)}}(\bar{t})=t$ существует в $H$ при правильном выборе единственного әлемента из множества $J_{1}=\left\{1, a_{1}\right.$, $\left.a_{1}^{\left(d_{l}\right)_{1}}, a_{1} \cdot a_{1}^{\left(d_{l}\right)_{1}}\right\}, l=\omega_{2}$, для проекиии $\varphi_{R_{1}^{(1)}}(\bar{t})$.

б) Пусть $t \in H_{n}, n>1$. Тогда әлемент $\bar{t}$ с проекииями

$$
\varphi_{R_{n}^{\left(2^{n}\right)}}(\bar{t})=t, \quad \varphi_{\Delta_{i} R_{n}^{\left(2^{n}\right)}}(\bar{t})=u_{I(t), n-i+1}, \quad i=\overline{2, n}
$$

существует в Н при правильном выборе единственного әлемента из множества $J_{n}=\left\{1, a_{n}, a_{n}^{\left(d_{l}\right)_{n}}, a_{n} \cdot a_{n}^{\left(d_{l}\right)_{n}}\right\}, l=\omega_{n+1}$, для проекиии $\varphi_{R_{n}^{\left(2^{n}-1\right)}}(\bar{t})$. 
ДокАЗАТЕЛЬСтво. Назовем $t$ главным по отношению $\kappa \bar{t}$, а элемент из множества $J_{n}$ побочным. Будем говорить, что $\bar{t}$ есть распространение әлемента $t$ уровня $n$ относительно интервала $R_{n}^{\left(2^{n}\right)}$. Доказательство существования распространения проведем индукцией по $n$. Будем считать, что $j \in \Phi$.

a) Пусть $n=1$. Если $t=\left(d_{j}\right)_{1}$, то положим $\bar{t}=d_{j}$, при этом $\varphi_{R_{1}^{(1)}}(\bar{t}) \in\left\{1, a_{1}\right\}$.

Аналогично, для $t=\left(d_{j}\right)_{1}^{a_{1}}$ возьмем $\bar{t}=d_{l}^{a} \cdot d_{j} \cdot d_{l}^{a}$, где $l=\omega_{2}$. В этом случае $\varphi_{R_{1}^{(1)}}(\bar{t}) \in\left\{1, a_{1}^{\left(d_{l}\right)_{1}}\right\}$. Однозначньй выбор $l$ возможен, поскольку $l \neq \omega_{1}$ и $\left|a_{1}\left(d_{l}\right)_{1}\right|=4$. Последнее равенство показывает, что побочные элементы порождающих элементов группы $H_{1}$ формируют множество $J_{1}$ и, тем самым, утверждение а) доказано.

б) Из предположения $\bar{t} \in G$ в силу леммы 1 , примененной к элементу $\varphi_{R_{n-1}^{\left(2^{n-1}\right)}}(\bar{t})$, и того, что характеристики элементов множества $J_{n}$ попарно различны, следует, что в множестве $J_{n}$ не может быть более одного побочного элемента для данного $t$.

Допустим, лемма доказана для $k<n$. Покажем существование распространения при $k=n$.

Равенство $\varphi_{\Delta_{i} R_{n}^{\left(2^{n}\right)}}(\bar{t})=u_{I(t), n-i+1}, i=\overline{2, n}$, следует из утверждения а) леммы 1 , примененной к элементу $\varphi_{\Delta_{i} R_{n}^{\left(2^{n}\right)} \cup \Delta_{1}\left(\Delta_{i} R_{n}^{\left(2^{n}\right)}\right)}(\bar{t})$.

Если $t=\left(d_{j}\right)_{n}$, то аналогично доказательству утверждения а) полагаем $\bar{t}=d_{j}$.

Пусть теперь $t=\left(\left(d_{j}\right)_{n}\right)^{a_{n}}$. Рассмотрим элемент $t_{1}=\left(\left(d_{l}\right)_{n-1}\right)^{a_{n-1}}$, где $l=\omega_{n+1}$, в качестве главного на интервале $R_{n-1}^{\left(2^{n-1}\right)}$. По нашему предположению на интервале $R_{n-1}^{\left(2^{n-1}-1\right)}$ для $t_{1}$ найдется побочньй элемент

$$
\begin{cases}1, & \text { если } l=\omega_{n-1}, \\ a_{n-1}^{\left(d_{\omega_{n}}\right)_{n-1},}, & \text { если } l \neq \omega_{n-1} .\end{cases}
$$

Непосредственно проверяется, что $\bar{t}=\left(d_{j}\right)^{\bar{t}_{1}}$ является искомьм распространением элемента $t$ уровня $n$, поэтому распространение порождающих группы $H_{n}$ найдено.

Рассмотрим гомоморфизм $z_{n}: H_{n} \rightarrow H$ с условием

$$
z_{n}\left(\left(d_{j}\right)_{n}\right)=d_{j}, \quad z_{n}\left(\left(\left(d_{j}\right)_{n}\right)^{a_{n}}\right)=\left(d_{j}\right)^{\bar{t}_{1}}
$$

Аналогично утверждению а) распространением $t \in H_{n}$ является элемент $z_{n}(t)$. Лемма доказана.

Будем считать $B_{n}^{(i)}$ меткой элемента $t$, если $\varepsilon\left(\varphi_{R_{n}^{(i)}}(t)\right)=1, n \in \mathbb{Z}_{+}, i=\overline{1,2^{n}}$. Пусть $M(t)$ - множество меток элемента $t$. Назовем метку $B_{n}^{(i)}$ свободной относительно $M(t)$, если $B_{n}^{(i)} \in M(t)$ и для любого другого элемента $B_{r}^{(s)} \in M(t)$ выполняется $R_{r}^{(s)} \cap R_{n}^{(i)}=\varnothing$.

ПРЕДЛОЖЕНИЕ 2. Для любой последовательности $\omega \in R \Omega_{0}$ и любого интервала $R_{n}^{(i)}$ существует $t \in G_{\omega}$ такое, что метка $B_{n}^{(i)}$ является свободной относительно $M(t)$. 
ДоКАЗАТЕЛЬСтво. Построим по индукции множество элементов $(i, n)_{\Pi}$ грушшы $G_{\omega}$, для которых метка $B_{n}^{(i)}$ является свободной относительно $M\left((i, n)_{\Pi}\right)$. Одновременно построим множество элементов $(i, n)_{T} \in G_{\omega}$ таких, что для любой метки $B_{r}^{(s)} \in$ $M\left((i, n)_{T}\right)$ выполняется $R_{r}^{(s)} \cap R_{n}^{(i)} \neq \varnothing$.

Возьмем $(1,1)_{\Pi}=d_{j},(2,1)_{\Pi}=\left(d_{j}\right)^{a}$, где $j \in \Phi \backslash\left\{\omega_{1}\right\}$, а также $(1,1)_{T}=d_{\omega_{1}}$, $(2,1)_{T}=\left(d_{\omega_{1}}\right)^{a}$.

Будем говорить, что $t$ на интервале $R_{n}^{(i)}$ имеет свободный активный порождающий, если $\varphi_{R_{n}^{(i)}}(t)=\left(d_{j}\right)_{n}, j \in \Phi$, и для любого $B_{r}^{(s)} \in M(t)$ такого, что $r \neq n$, вьполняется $R_{n}^{(i)} \cap R_{r}^{(s)}=\varnothing$. Построенные нами $(i, 1)_{\Pi \text { и }}(i, 1)_{T}$ имеют свободные активные порождающие на интервале $\Delta_{1} R_{1}^{(i)}, i=1,2$.

Пусть уже построены элементы $(i, k)_{\Pi}$ и $(i, k)_{T}$ для $k<n, i=\overline{1,2^{k}}$, которые имеют свободные активные порождающие на интервале $\Delta_{1} R_{k}^{(i)}$. Построим $(i, n)_{\Pi \text { и }}(i, n)_{T} \mathrm{c}$ аналогичньм свойством, $i=\overline{1,2^{n}}$.

Для каждого $i=\overline{1,2^{n}}$ определим $m$ из включения $R_{n}^{(i)} \subset \Delta_{1} R_{n-1}^{(m)}$. Если $i$ нечетно, то возьмем

$$
(i, n)_{\Pi}= \begin{cases}(m, n-1)_{T}, & \text { если } \omega_{n-1} \neq \omega_{n}, \\ (m, n-1)_{\Pi}, & \text { если } \omega_{n-1}=\omega_{n} .\end{cases}
$$

Пусть теперь $i$ четно. Положим $(i, n)_{\Pi}=\left((i-1, n)_{\Pi}\right)^{(i / 2, n-1)_{\Pi}}$. Для определенности будем считать, что $\omega_{n-1} \neq \omega_{n}$. Тогда по предположению индукции

$$
\begin{aligned}
\varphi_{R_{n-1}^{(i / 2)}}\left((i, n)_{\Pi}\right) & =\left(\varphi_{R_{n-1}^{(i / 2)}}\left((m, n-1)_{T}\right)\right)^{a_{n-1}} \\
& =\left(\varphi_{\Delta_{1} R_{n-1}^{(m)}}\left((m, n-1)_{T}\right)\right)^{a_{n-1}}=\left(\left(d_{\omega_{n-1}}\right)_{n-1}\right)^{a_{n-1}} .
\end{aligned}
$$

Отсюда следует, что $\varphi_{R_{n}^{(i)}}\left((i, n)_{\Pi}\right)=u_{n, \omega_{n-1}}=a_{n}$ и $\varphi_{\Delta_{1} R_{n}^{(i)}}\left((i, n)_{\Pi}\right)=\left(d_{\omega_{n-1}}\right)_{n}$, т.е. так выбранный элемент $(i, n)_{\Pi}$ имеет свободньй активньй порождаюший на интервале $\Delta_{1} R_{n}^{(i)}$. Случай $\omega_{n-1}=\omega_{n}$ рассматривается аналогично. Осталось отметить, что элемент $(i, n)_{T}$ строится таким же образом. Предложение доказано.

Следуюшие результаты демонстрируют роль групшы характеристик для $G=G_{\omega}$, $\omega \in R_{1} \Omega_{0}$.

Лемма 3. Пусть $t_{1}, \ldots, t_{2^{n}} \in G_{n}, n \in \mathbb{N} . \quad E c л u \chi_{n}\left(t_{i}\right)=\left(d_{0}, 0,0\right)$, где $d_{0}-$ единица группы $\mathbb{Z}_{2} \times \mathbb{Z}_{2}$, для любого $i=\overline{1,2^{n}}$, то существует $t \in H^{(n+1)}$ с условием $\varphi_{R_{n}^{(i)}}(t)=t_{i}$

ДокАЗАТЕЛЬСТво. Пусть $t \in G_{n}, \chi_{n}(t)=\left(d_{0}, 0,0\right)$. Обозначим через $t(i)$ элемент группы $P_{\infty}$ с условиями $\varphi_{R_{n}^{(s)}}(t(i))=1$ при $s \neq i, \varphi_{R_{n}^{(i)}}(t(i))=t, i \in \overline{1,2^{n}}$. Покажем, что $t(i) \in G$. При $i=2^{n}$ это следует из леммы 2 . Если $i=\overline{1,2^{n}-1}$, то $t(i)$ можно получить из $t\left(2^{n}\right)$ посредством сопряжений подходящими элементами $(m, n)_{\Pi}$. Точнее, если

$$
i=2^{n}-\left(2^{n-k}+\rho_{k-1} 2^{n-k-1}+\cdots+\rho_{1} 2^{n-1}\right), \quad \rho_{1}, \ldots, \rho_{k-1} \in\{0,1\},
$$

TO

$$
t(i)=\left(t\left(i+2^{n-k}\right)\right)^{\left(2^{n-k-1}, n-k-1\right)_{\Pi}} \quad \text { и } \quad t\left(2^{n}-2^{n-1}\right)=\left(t\left(2^{n}\right)\right)^{a} .
$$


Нетрудно видеть, что $t=t_{1}(1) \cdots t_{2^{n}}\left(2^{n}\right)$.

Назовем $t(i)$ распространением әлемента $t$ относительно интервала $R_{n}^{(i)}$.

СлЕДСтвиЕ. Пусть $t \in H \quad u \chi_{n}\left(\varphi_{R_{n}^{(i)}}(t)\right)=\chi_{n, i} \in L_{\omega_{n}}, i=\overline{1,2^{n}}$. Тогда для любой последовательности $g_{i} \in G_{n}$ с характеристиками $\chi_{n}\left(g_{i}\right)=\chi_{n, i}$ существует $g \in H$, имеющее гранииу $\partial_{n}(g)=\left(g_{1}, \ldots, g_{2^{n}}\right)$.

ДокаЗАтельство. Положим $t_{i}=\varphi_{R_{n}^{(i)}}(t)$. Рассмотрим элемент $\bar{g} \in P_{\infty}$ со схемой $s_{n}(\bar{g})=\left(g_{1} t_{1}^{-1}, \ldots, g_{2^{n}} t_{2^{n}}^{-1} ; 1\right)$. По лемме 3 выполняется $\bar{g} \in H^{(n+1)}$. Ясно, что $g=\bar{g} t \in H$.

Итак, если любую проекцию $n$-го уровня элемента групшы $G$ заменить на другой элемент с сохранением характеристики, то новый элемент также принадлежит $G$. Последовательность элементов $\chi_{n}^{(1)}, \ldots, \chi_{n}^{\left(2^{n}\right)}$ групшы $L_{\omega_{n}}$ назовем правильной, если в $G$ существует хотя бы один элемент $g$ с условием $\chi_{n}\left(\varphi_{R_{n}^{(i)}}(g)\right)=\chi_{n}^{(i)}$.

Для $n \geqslant 0$ рассмотрим множество $\operatorname{In}_{n}(G)$, состоящее из элементов $g \in P_{\infty}$, для которых все проекции $\varphi_{R_{n}^{(i)}}(g)$ принадлежат $G_{n}$. Очевидно, $\operatorname{In}_{n}(G) \subseteq \operatorname{In}_{n+k}(G), k \in \mathbb{N}$, и $\operatorname{In}_{0}(G)=G$.

Следуюшее утверждение является в некотором смысле обрашением леммы 1.

Лемма 4. Пусть $g \in \operatorname{In}_{n}(g), n \in \mathbb{N}$. Тогда $g \in \operatorname{In}_{n-1}(G)$, если и только если последовательность $\chi_{n, i}=\chi\left(\varphi_{R_{n}^{(i)}}(g)\right)=\left(d_{j_{i}}, \varepsilon_{i}, \tau_{i}\right) \in L_{\omega_{n}}, i=\overline{1,2^{n}}$, подчинена условиям

$$
\varepsilon_{i}=Q\left(j_{\delta(i)},\left\{0, \omega_{n}\right\}\right), \quad \tau_{i}+\tau_{\delta(i)}=\varepsilon_{i} \cdot \varepsilon_{\delta(i)} .
$$

ДокАЗАТЕЛЬСтво. Необходимость следует из леммы 1 , примененной к проекциям.

Доказывать достаточность будем индукцией по $n$ сразу для всех $\omega \in R_{1} \Omega_{0}$. Пусть $g \in \operatorname{In}_{1}\left(G_{\omega}\right)$ и верно (3). Покажем, что $g \in G_{\omega}$.

Не ограничивая общности, считаем $B_{0} \notin M(g), \varphi_{R_{1}^{(1)}}(g) \in H_{\sigma \omega}$. Если это не так, то элемент $g$ приводится к необходимому виду умножением на $d_{j}$ и (или) на $a, j \neq \omega_{1}$.

Положим $g_{i}=\varphi_{R_{1}^{(i)}}(g), \chi_{1}\left(g_{i}\right)=\left(d_{j_{i}}, \varepsilon_{i}, \tau_{i}\right)=\chi_{i}, i=1,2$. Следуя лемме 2 , построим $g^{\prime}$ - распространение элемента $g_{1}$ относительно $R_{1}^{(1)}$. При этом $\varphi_{R_{1}^{(2)}}\left(g^{\prime}\right)=g_{2}^{\prime} \in J_{1}$ и $\chi_{1}\left(g_{2}^{\prime}\right)=\left(d_{0}, Q\left(j_{1},\left\{0, \omega_{1}\right\}\right), \tau_{1}\right)$.

В то же время из условий (3) в силу выбора $\varepsilon_{1}$ имеем $j_{2} \in\left\{0, \omega_{1}\right\}, \varepsilon_{2}=Q\left(j_{1},\left\{0, \omega_{1}\right\}\right)$, $\tau_{2}=\tau_{1}$.

Рассмотрим $g^{\prime \prime}$ - распространение $\left(g_{2}^{\prime}\right)^{-1} g_{2}$ относительно интервала $R_{1}^{(2)}$. Так как $\chi_{1}\left(\left(g_{2}^{\prime}\right)^{-1} g_{2}\right)=\left(\chi_{1}\left(g_{2}^{\prime}\right)\right)^{-1} \cdot \chi_{1}\left(g_{2}\right)=\left(d_{j_{2}}, 0,0\right)$, по лемме $2 g^{\prime \prime}$ можно взять с условием $\varphi_{R_{1}^{(1)}}\left(g^{\prime \prime}\right)=1$. Итак, $g^{\prime}, g^{\prime \prime} \in G_{\omega}$ и $g=g^{\prime} g^{\prime \prime}$.

Предположим теперь $g \in \operatorname{In}_{n}\left(G_{\omega}\right)$ и выполнено (3). Из доказанной достаточности для случая $\operatorname{In}_{1}\left(G_{\omega}\right)$, применяя рассуждение к групше $G_{\sigma^{n-1} \omega}$, получаем, что

$$
\varphi_{R_{n-1}^{(i)}}(g) \in G_{\sigma^{n-1} \omega}, \quad g \in \operatorname{In}_{n-1}\left(G_{\omega}\right) .
$$

ЗАмЕчАНИЕ. Лемма 4 позволяет строить все правильные последовательности уровня $n$ элементов грушшы $L_{\omega_{n}}, n \geqslant 1$. 
3. Критерий сопряженности. Пусть $g, h$ сопряжены в $G$, т.е. для некоторого $t \in G$ вьполняется $t^{-1} g t=h$. Положим $n=\max (f(g), f(h), 1)$,

$$
g_{i}=\varphi_{R_{n+1}^{(i)}}(g), \quad h_{i}=\varphi_{R_{n+1}^{(i)}}(h), \quad t_{i}=\varphi_{R_{n+1}^{(i)}}(t), \quad i=\overline{1,2^{n+1}} .
$$

Очевидно, что $\beta^{-1} g^{(n)} \beta=h^{(n)}$, где $\beta=t^{(n)} \in K^{(n)}$. По правилу получения схемы произведения, имеем $\left(t^{-1}\right)_{i} g_{t^{(n)}{ }_{i}} t_{\left(g^{(n)}\right)^{-1} t^{(n)} i}=h_{i}$, или при $k=t^{(n)} i$

$$
g_{k} t_{\left(g^{(n)}\right)^{-1} k}\left(h_{\left(t^{(n)}\right)^{-1} k}\right)^{-1}=t_{k} .
$$

Очевидно,

$$
g_{k} g_{\left(g^{(n)}\right)^{-1} k} t_{\left(g^{(n)}\right)^{-2} k}\left(h_{\left(t^{(n)}\right)^{-1}\left(g^{(n)}\right)^{-1} k}\right)^{-1}\left(h_{\left(t^{(n)}\right)^{-1} k}\right)^{-1}=t_{k} .
$$

Пусть порядок $g^{(n)}$ равен $U$. Тогда

$$
\left(\prod_{q=0}^{U-1} g_{\left(g^{(n)}\right)^{-q} k}\right) t_{k}\left(\prod_{q=0}^{U-1} h_{\left(t^{(n)}\right)^{-1}\left(g^{(n)}\right)^{-q} k}\right)^{-1}=t_{k} .
$$

Элементы в скобках обозначим через $g_{n}(k, \beta)$ и $h_{n}(k, \beta)$ соответственно. Это определение корректно для $h_{n}(k, \beta)$, поскольку $\left(t^{(n)}\right)^{-1}\left(g^{(n)}\right)^{-q} k=\left(h^{(n)}\right)^{-q}\left(t^{(n)}\right)^{-1} k$.

Мы получаем условие на элемент $t_{k}$ :

$$
\left(t_{k}\right)^{-1} g_{n}(k, \beta) t_{k}=h_{n}(k, \beta), \quad k=\overline{1,2^{n+1}} .
$$

Лемма 5. Имеют место оценки

$$
\operatorname{len}\left(g_{2 n+1}\left(k, t^{(2 n)}\right)\right) \leqslant 1, \quad \operatorname{len}\left(h_{2 n+1}\left(k, t^{(2 n)}\right)\right) \leqslant 1, \quad k=\overline{1,2^{2 n+1}} .
$$

ДокАЗАТЕЛЬСТВО. Неограничивая обшности, проведем доказательство только для $g_{2 n+1}\left(k, t^{(2 n)}\right)$. Заметим, что для любых $v, x \in G$ справедливо следующее равенство, дающее большую информацию о роли действия элемента конечного сплетения на множество:

$$
\varphi_{R_{n+r}^{(x+n+r-1) \bar{k})}}(v)=\varphi_{R_{r}^{(\tilde{k})}}\left(\varphi_{R}(v)\right),
$$

где $\bar{k}=(k-1) 2^{r}+i, \tilde{k}=\left(\varphi_{R}(v)\right)^{(r-1)} i, R=R_{n}^{\left(x^{(n-1)} k\right)}, i=\overline{1,2^{r}}, k=\overline{1,2^{n}}, r \in \mathbb{N}$.

Обозначим проекцию слева через $v_{n+r}(\bar{k}, x)$.

Пусть $f(v) \leqslant n+r-1$. Если $v_{n+r}(\bar{k}, x)=a_{n+r}$, то

$$
v_{n+r-1}\left(k^{\prime}, x\right)=\left(d_{j}\right)_{n+r-1}, \quad j \neq \omega_{n+r}, \quad \bar{k} \in\left\{2 k^{\prime}-1,2 k^{\prime}\right\},
$$

и

$$
v_{n+r+1}(\overline{\bar{k}}, x)=1, \quad \overline{\bar{k}} \in\{2 \bar{k}, 2 \bar{k}-1\} .
$$

Так как длина len $\left(g_{n+1}\left(\bar{k}, t^{(n)}\right)\right) \leqslant U \leqslant 2^{n}$ при $\bar{k}=\overline{1,2^{n+1}}$, то, выбирая для $q=\overline{1, U}$ элементы $x=g^{-q}, v=g, r=1$ и используя $\left(g^{-q}\right)^{(r)}=\left(g^{(r)}\right)^{-q}$, очевидно, получаем

$$
\operatorname{len}\left(g_{n+2}\left(\overline{\bar{k}}, t^{(n+1)}\right)\right) \leqslant\left[\frac{U+1}{2}\right] .
$$

Отсюда по индукции $\operatorname{len}\left(g_{2 n+1}\left(k, t^{(2 n)}\right)\right) \leqslant 1$ для любого $k=\overline{1,2^{2 n+1}}$.

Из леммы 5 и (4) следует, что в случае $t^{-1} g t$ выполняются равенство $g_{2 n+1}\left(k, t^{(2 n)}\right)=$ $h_{2 n+1}\left(k, t^{(2 n)}\right)$ и вхождение $t_{k} \in C_{G_{2 n+1}}\left(g_{2 n+1}\left(k, t^{(2 n)}\right)\right)$ для любого $k=\overline{1,2^{2 n+1}}$.

Докажем основной результат. 
ТеоремА (критерий сопряженности). Пусть $\omega \in R_{1} \Omega_{0}, g, h \in G_{\omega} u m=2 \max (f(g)$, $f(h), 1)+3$. Если $g^{(m)}$ и $h^{(m)}$ сопряхсень в $K_{\omega}^{(m)}$, то $g$ и $h$ сопряхсены в $G_{\omega}$. Кроме того, существует $t \in G_{\omega}$ с условием $g^{t}=h$ такое, что $f(t) \leqslant m$.

ДокАЗАТЕЛЬСтво. Сохраняя обозначения этого пункта, выберем $\beta \in K_{\omega}^{(m)}$, для которого справедливо

$$
\left(g^{(m)}\right)^{\beta}=h^{(m)} .
$$

В группе $K_{\omega}^{(m)}$ проблема сопряженности разрешима, так как $K_{\omega}^{(m)}$ является подгруппой сплетения $P_{m+1}$ с явно известньми порождающими (формула (1) для первых $m+1$ координат).

Назовем $t \in G_{\omega}$ продолэсением $\beta$, если $t^{(m)}=\beta$. Заметим, что хотя бы одно продолжение мы можем установить всегда. Действительно, представим $\beta$ в виде произведения порождающих групшы $K_{\omega}^{(m)}$. Тем самым, $\beta=(a) d_{j_{1}}^{(m)} a \cdots a d_{j_{\mu}}^{(m)}(a)$ и $t=(a) d_{j_{1}} a \cdots$ $a d_{j_{\mu}}(a)$ - одно из продолжений. Нетрудно видеть, что любое другое продолжение $t^{\prime}$ представимо в виде $\alpha t$, где $\alpha \in H_{\omega}^{(m+1)}$. Из (5) следует, что

$$
t_{\left(g^{(m-3)}\right)^{-1} k}^{(2)}=\left(g_{k}^{(2)}\right)^{-1} \cdot t_{k}^{(2)} \cdot h_{\beta^{-1} k}^{(2)}, \quad k=\overline{1,2^{m-2}}
$$

Пользуясь леммой 1 и неравенством $\omega_{s+1} \neq \omega_{s+2}$, несложно установить, что характеристика $\chi_{s}$ любого элемента $v \in G_{\sigma^{s}} \omega$ может быть определена однозначно по элементу $v^{(2)} \in K_{\sigma^{s} \omega}^{(2)}$. Отсюда

$$
\chi_{m-2}\left(t_{\left(g^{(m-3)}\right)^{-1} k}\right)=\chi_{m-2}\left(g_{k}^{-1}\right) \cdot \chi_{m-2}\left(t_{k}\right) \cdot \chi_{m-2}\left(h_{\beta^{-1} k}\right) .
$$

Это равенство дает возможность использовать идею п. 2 - формировать необходимьй нам элемент $\tilde{t}$ путем замены на уровне $m-2$ существующих проекций иными элементами $\tilde{t}_{k}$ групшы $G_{\sigma^{m-2} \omega}$ с сохранением последовательности характеристик проекций.

Пусть $i_{1}, \ldots, i_{\zeta}$ - представители различных орбит множества $\left\{1, \ldots, 2^{m-2}\right\}$, порожденных элементом $\left(g^{(m-3)}\right)^{-1}$. Будем требовать, чтобы $t_{i_{r}}$ принадлежал централизатору $C_{G_{\sigma m-2}{ }_{\omega}}\left(g_{m-2}\left(i_{r}, \tilde{\beta}\right)\right)$, где $\tilde{\beta}=t^{(m-3)}, r=1, \ldots, \zeta$. Если теперь $\tilde{\beta}^{-1} i \neq i_{r}$, $r \in \overline{1, \zeta}$, и вьполняется

$$
\tilde{t}_{\tilde{\beta}^{-1} i}=\left(g_{i}\right)^{-1} \cdot \tilde{t}_{i} \cdot h_{\tilde{\beta}^{-1} i},
$$

то элемент $\tilde{t}$ со схемой $s_{m-2}(\tilde{t})=\left(\tilde{t}_{1}, \ldots, \tilde{t}_{2^{m-2}} ; \tilde{\beta}\right)$ будет переводить $g$ в $h$ согласно (4). При этом $\tilde{t}$ принадлежит $G_{\omega}$ в силу правильности последовательности характеристик его проекций.

Осталось обосновать возможность выбора $\tilde{t}_{k}$. По правилу произведения элементов групп $L_{y}, y \in \Phi$, видно, что $C_{G_{\sigma m-2}}\left(a_{m-2}\right)$ не содержит элементов индекса отличного от 0. Аналогично, если $\xi \in C_{G^{m-2} \omega}\left(\left(d_{j^{\prime}}\right)_{m-2}\right), j^{\prime} \in \Phi$, то

$$
\chi_{m-2}(\xi) \in\left\{\begin{array}{l}
\left\{\left(d_{j}, 0,0\right)\right\}, \\
\left\{\left(d_{j}, 0, \tau\right)\right\}, \\
\text { если } j^{\prime} \neq \omega_{m-3},
\end{array} \quad \tau \in \mathbb{Z}_{2}, \quad j \in \Phi\right.
$$


Для каждой возможной характеристики централизаторов порождающих группы $G_{\sigma^{m-2} \omega}$ находим конкретньй элемент:

\begin{tabular}{|c|c|}
\hline характеристика $\chi_{m-2}$ & элемент централизатора \\
\hline$\left(d_{0}, \varepsilon, \tau\right)$ & $\left(\left(d_{\eta}\right)_{m-2}^{a_{m-2}}\left(d_{\eta}\right)_{m-2}\right)^{\tau} a_{m-2}^{\varepsilon}$ \\
\hline$\left(d_{j}, 0,1\right)$ & $\left(d_{\eta}\right)_{m-2}^{a_{m-2}}\left(d_{\eta} d_{j}\right)_{m-2}$ \\
\hline$\left(d_{j}, 0,0\right)$ & $\left(d_{j}\right)_{m-2}$ \\
\hline
\end{tabular}

где $\eta=\omega_{m-3}$.

Для $\tilde{t}_{i_{1}}, \ldots, \tilde{t}_{i_{\zeta}}$ выбираем элементы из данной таблицы по требованию характеристики, а остальные $\tilde{t}_{i}$ получаем при помощи (6).

Таким образом, мы построили $\tilde{t} \in G_{\omega}$ с условием $g^{\tilde{t}}=h$. Заметим, учитьвая вид $g_{i}$ и $h_{\tilde{\beta}^{-1} i}$, что все элементы $\tilde{t}_{i}$ имеют ранг не больше 2 . Отсюда $f(\tilde{t}) \leqslant m$.

Из доказанной теоремы вытекает два возможных критерия сопряженности. Во-первых, элементы $g, h \in G_{\omega}$ для $\omega \in R_{1} \Omega_{0}$ сопряжены, если сопряжены $g^{(2 n+3)}$ и $h^{(2 n+3)}$ при $n=\max (f(g), f(h), 1)$. Отсюда разрешимость проблемы сопряженности в группе $G_{\omega}$ следует из ее разрешимости в группах $K_{\omega}^{(m)}, m \in \mathbb{N}$. Это означает, что групша $G_{\omega}$ является конечной относительно сопряженности.

Во-вторьх, если $g$ и $h$ сопряжены, то найдется элемент $t$ ограниченного ранга, которьй удовлетворяет равенству $g^{t}=h$. В этом случае сопряженность $g$ и $h$ выясняется ввиду возможности перебора всех элементов, имеющих ранг ниже некоторого фиксированного.

Отметим, что леммы 1-4 не зависят от проблемы сопряженности. По-видимому, идеи, заложенные в них, могут оказаться действенньги и при изучении других свойств группы $G_{\omega}$, а также при изучении $p$-групп Григорчука, $p$-групп Гупты и Сидки [4] и других периодических групп похожей конструкции.

В заключение отметим, что совсем недавно Дж. Вилсон и П. Залесский [5] показали конечность относительно сопряженности $p$-групп Григорчука и $p$-групп Гупты и Сидки, где $p$ - нечетное простое. Удовлетворяют ли условию их основной теоремы 2-групшы Григорчука по-прежнему неизвестно, хотя следствие, как видно из результатов данной работы, является верньм.

Автор выражает глубокую признательность В. И. Сушанскому и М. Н. Васякиной за их поддержку и внимание к работе.

\section{СПИСОК ЦИТИРОВАННОЙ ЛИТЕРАТУРЫ}

[1] Григорчук Р.И. К проблеме Бернсайда о периодических группах // Функцион. анализ и его прилож. 1980. Т. 14. № 1. С. 53-54.

[2] Григорчук Р. И. Степени роста конечно порожденных групп и теория инвариантных средних // Изв. АН СССР. Сер. матем. 1984. №5. С. 939-985.

[3] Сущанский В. И. Сплетения и периодические факторизуемые группы // Матем. сб. 1989. T. 180. № 8. C. 1073-1091.

[4] Gupta N., Sidki S. Some infinite $p$-groups // Алгебраи логика. 1983. T. 22. № 5. С. 584-589.

[5] Wilson J.S., Zalesskii P. A. Conjugacy separability of certain torsion groups // Arch. Math. (Basel). 1997. V. 68. P. 441-449. 